\title{
Factores pronósticos de mortalidad intrahospitalaria en pacientes con infarto agudo del miocardio con elevación del segmento ST. Hospital Arnaldo Milián Castro. Villa Clara, Cuba. 2015
}

\author{
David I. Padilla-Cueto* \\ Halbert Hernández-Negrín** \\ Arlenys Pérez-Valdivia*** \\ Eligio Barreto-Fiu***** \\ José I. Ramírez-Gómez ${ }^{* * * * *}$
}

\begin{abstract}
*Estudiante de VI año de Medicina. Alumno Ayudante en Cardiología. Hospital Arnaldo Milián Castro. Universidad de Ciencias Médicas de Villa Clara. Villa Clara. Cuba.

**Residente de II año de Medicina Interna. Servicio de Medicina Interna. Hospital Arnaldo Milián Castro. Universidad de Ciencias Médicas de Villa Clara. Villa Clara. Cuba.

***Residente de Il año de Microbiología. Laboratorio de Microbiología. Hospital Arnaldo Milián Castro. Universidad de Ciencias Médicas de Villa Clara. Villa Clara. Cuba.

****Máster en Computación Aplicada. Profesor Auxiliar. Universidad de Ciencias Médicas de Villa Clara. Villa Clara. Cuba.

*****Especialista en Medicina General Integral y Cardiología. Jefe de Servicio de Cardiología. Hospital Universitario Arnaldo Milián Castro. Universidad de Ciencias Médicas de Villa Clara. Villa Clara. Cuba.

Correspondencia: Sr. Padilla-Cueto. Calle A \#29, entre C. Camajuaní y Circunvalación, Santa Clara, Villa Clara, Cuba. Código Postal: 50300. Correo electrónico: davidpadillacueto@gmail.com
\end{abstract}

\section{RESUMEN}

Introducción: establecer los factores relacionados con el incremento de la mortalidad en pacientes con infarto agudo del miocardio contribuye a diferenciar su seguimiento y facilita la elección de conductas específicas que determinan mejor evolución. Objetivo: identificar los factores pronósticos de mortalidad intrahospitalaria en pacientes con infarto agudo del miocardio con elevación del segmento ST. Métodos: se realizó un estudio analítico de tipo casos y controles en pacientes admitidos con infarto agudo del miocardio con elevación del segmento ST en el Hospital Arnaldo Milián Castro durante el 2015. El grupo caso quedó conformado por todos los pacientes fallecidos $(n=15)$ y el grupo control por pacientes vivos $(n=60)$. Se evaluaron durante el ingreso variables epidemiológicas, hemodinámicas y de laboratorio y se realizó regresión logística binaria para identificar los factores pronósticos. Resultados: la media de la edad (74.13 \pm 7.80 ),

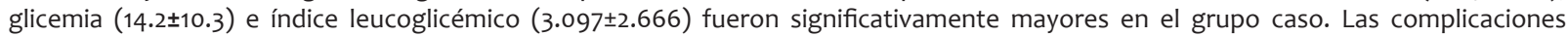
eléctricas (OR:2.00; IC:1.767 a 20.369) y hemodinámicas (OR:2.00; IC:1.398 a 2.860) predominaron en los pacientes fallecidos. El análisis multivariado identificó como predictores de mortalidad a la creatinina (OR:1.013; IC:1.010 a 1.027), el tratamiento de reperfusión coronaria (OR:0.206; IC:0.049 a 0.868) y el Killip-Kimball>I (OR:8.311; IC:1.511 a 45.720). Conclusiones: los niveles séricos de creatinina, el tratamiento de reperfusión coronaria y la clasificación Killip-Kimball>I constituyeron factores pronósticos de mortalidad intrahospitalaria en pacientes villaclareños con infarto agudo del miocardio con elevación del segmento ST. Su identificación temprana podría indicar qué pacientes necesitan un seguimiento más estrecho durante su estadía hospitalaria para mejorar su pronóstico. MÉD.UIS. 2017;30(3):67-74.

Palabras Clave: Infarto del miocardio. Mortalidad hospitalaria. Pronóstico.

\section{Prognostic factors of hospital mortality in patients with ST elevation myocardial infarction. Hospital Arnaldo Milián Castro. Villa Clara, Cuba. 2015}

\section{ABSTRACT}

Introduction: establishing the adequate prognosis in patients with acute myocardial infarction contributes to differentiate their follow-up and facilitates when deciding which specific conduct will determine the best result in its course. Objective: to identify prognostic factors of hospital mortality in patients with ST elevation myocardial infarction. Methods: it was performed a case-control analytic study in patients 
admitted with ST elevation myocardial infarction in "Arnaldo Milián Castro" hospital during 2015. The case group was integrated by deceased patients $(n=15)$ and the control group by living patients $(n=60)$. During admission, demographic, hemodynamic and laboratory variables were evaluated, and a binary logistic regression was made to identify the predicting factors. Results: medium age (74.13 \pm 7.80$)$,

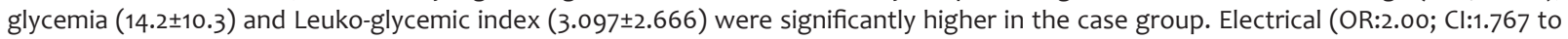
20.369) and hemodynamic complications (OR:2.00; Cl:1.398 a 2.860) predominated in deceased patients. Multivariate analysis identified serum creatinine (OR:1.013; Cl:1.010 to 1.027), coronary reperfusion therapy (OR:0.206; Cl:0.049 to 0.868) and Killip-Kimball classification (OR:8.311; Cl:1.511 to 45.720) as prognostic factors of mortality. Conclusions: the serum creatinine levels, coronary reperfusion therapy and Killip-Kimball classification constituted the prognostic factors of hospital mortality for patients with ST elevation myocardial infarction. An early identification of those factors could indicate that patients need a closer follow-up during admission to improve their prognosis. MÉD. UIS. 2017;30(3):67-74.

Key Words: Myocardial infarction. Hospital mortality. Prognosis.

¿Cómo citar este artículo?: Padilla-Cueto DI, Hernández-Negrín H, Pérez-Valdivia A, Barrreto-Fiu E, Ramírez-Gómez Jl. Factores pronósticos de mortalidad intrahospitalaria en pacientes con infarto agudo del miocardio con elevación del segmento ST. Hospital Arnaldo Milián Castro. Villa Clara, Cuba. 2015. MED.UIS. 2017;30(3):67-74.

\section{INTRODUCCIÓN}

Las enfermedades cardiovasculares han incrementado progresivamente su incidencia en todo el mundo de tal modo que han llegado a ser la primera causa de mortalidad; dentro de ellas, el Infarto Agudo del Miocardio (IAM) posee un lugar protagónico'.

Globalmente, el número de muertes por enfermedades cardiovasculares aumentó en un $30 \%$ entre 1990 y 2015; reportándose alrededor de 17.92 millones de fallecidos en 2015, cifra que se espera que se eleve hasta 23.6 millones en el $2030^{1}$. En Latinoamérica y el Caribe en 2015, causaron el $28.8 \%$ de todas las defunciones en la región, con una tasa de mortalidad de 280 personas por cada 100 000 habitantes, de las cuales más del 50\% debido a cardiopatía isquémica'.

En Cuba, a pesar de la introducción de la estreptoquinasa y del sistema integrado de urgencias médicas en la década de los noventa, el IAM sigue teniendo un notable impacto en la salud de la población ${ }^{2}$. Ejemplo de ello es que en el año 2016 más de 36000 personas murieron por enfermedades cardiovasculares, de las cuales 16 157 fueron asociadas a cardiopatía isquémica. En el mismo periodo de tiempo, en Villa Clara, fallecieron por enfermedades cardiovasculares 2716 personas, constituyendo, al igual que en el país, la primera causa de muerte 3 .

Numerosas particularidades clínicas y paraclínicas pueden modificar la fisiopatología, el curso clínico y el pronóstico del IAM a través de diversos mecanismos.
Esta información es especialmente útil porque se puede obtener en la primera evaluación del paciente con métodos sencillos, económicos y accesibles; de esta manera se facilita la elección de la estrategia y el modo específico de tratamiento según el pronóstico anticipado de cada paciente.

Los factores esenciales en el pronóstico a corto plazo del IAM son el tamaño del infarto, la masa miocárdica que previamente no era funcional y las complicaciones que puedan modificar su curso clínico de forma aguda. También tienen valor pronóstico otros parámetros al ingreso cuya alteración se asocia, por lo general, a un mayor tamaño del infarto y afectación concomitante de otros sistemas del organismo como es la glicemia, azoemia, perfil lipídico y proteico, reactantes de fase aguda, entre otros. La predicción es, por tanto, de naturaleza multivariante ${ }^{4}$.

A nivel internacional y nacional se han realizado investigaciones con el objetivo de identificar factores pronósticos de mortalidad intrahospitalaria; sin embargo, los estudios realizados en la provincia Villa Clara, excepto uno ${ }^{5}$, se han limitado a identificarfactores asociados de manera aislada, sin utilizar las ventajas que ofrece la aplicación de un análisis multivariado ${ }^{6-8}$. Por lo tanto, se propone como objetivo, identificar los factores pronósticos de mortalidad intrahospitalaria en pacientes con infarto agudo del miocardio con elevación del segmento ST (IAMCEST) atendidos en el Hospital Arnaldo Milián Castro.

\section{Materiales y mÉtodos}

Diseño, población y muestra: se realizó un estudio retrospectivo analítico de tipo casos y controles en pacientes que fueron admitidos en el del Hospital 
Arnaldo Milián Castro con diagnóstico de IAMCEST según los criterios de la Tercera Definición Universal del infarto de miocardio ${ }^{9}$ en el período comprendido entre enero y diciembre de 2015. La asistencia médica hospitalaria fue brindada por cardiólogos e intensivistas en las salas de cuidados intensivos coronarios y de terapia intensiva de la institución de acuerdo a los protocolos establecidos.

La población estuvo constituida por 92 pacientes. El grupo caso quedó conformado por todos los pacientes fallecidos durante el ingreso $(n=15)$ y el grupo control por 60 pacientes que egresaron vivos, seleccionados al azar a través de un muestreo aleatorio simple. Se escogió este tamaño muestral en el grupo control para garantizar la existencia de una relación 1:4 entre ambos grupos con el objetivo de incrementar la solidez de los resultados y la potencia estadística del estudio ${ }^{10}$.

Variables: todas las variables fueron evaluadas durante el ingreso. Como variables independientes: sexo, color de la piel, edad (años cumplidos), antecedentes patológicos personales (obesidad, hábito de fumar, hipertensión arterial, hiperlipidemia, diabetes mellitus, alcoholismo, enfermedad vascular periférica, enfermedad cerebrovascular, IAM previo, insuficiencia renal crónica), variables hemodinámicas al ingreso (tensión arterial sistólica, tensión arterial diastólica, frecuencia cardíaca, clasificación Killip-Kimball'11), topografía del IAM según electrocardiograma, complementarios en las primeras 24 horas de ingreso: hematocrito, glicemia (mmol/L), leucocitos (10\%/L), neutrófilos, linfocitos, creatinina $(\mu \mathrm{mol} / \mathrm{L})$, índice leucoglicémico $\left(10^{\%} / \mathrm{L}^{*} \mathrm{mg} /\right.$ $\left.\mathrm{dL}^{*} 0.001\right)^{12}$ y pico máximo de creatininfosfoquinasa (U/L), tratamiento de reperfusión coronaria, complicaciones cardiovasculares (mecánicas, eléctricas, hemodinámicas, isquémicas, trombóticas e inflamatorias) y otras no cardiovasculares (trastornos del medio interno, neumonías o bronconeumonías, bacteriemia, descompensación de enfermedades no cardiovasculares, etc.). El estado al egreso (vivo/fallecido) fue seleccionado como variable dependiente o de respuesta.

Análisis estadístico y procesamiento de la información: los datos obtenidos de las historias clínicas fueron almacenados y procesados en el paquete estadístico SPSS versión 21 para Windows. Para caracterizar la muestra según variables de interés se usaron frecuencias absolutas y relativas expresadas en número y porcentaje. Para determinar las diferencias entre los grupos establecidos, según variables cualitativas, se usó el test estadístico $\mathrm{Chi}^{2}$ basado en su hipótesis de homogeneidad y el test exacto de Fisher según correspondiera. En el caso de las variables cuantitativas, se utilizaron medidas de resumen como media y Desviación Estándar (DE). Las medias fueron comparadas a través de la prueba t de Student de comparación de medias para grupos independientes en aquellas variables que presentaron una distribución normal (fue probada la normalidad a través de la prueba de Shapiro Wilk). Las variables cuantitativas que no se distribuyeron normalmente fueron comparadas a través del test de Mann Whitney.

Se determinó el Odd Ratio (OR) y sus Intervalos de Confianza (IC) para cada uno de los factores considerados como variables independientes de mortalidad. Además, se realizó una regresión logística binaria con el objetivo de determinar las variables que constituyen factores pronósticos de mortalidad. En este análisis multivariante fueron incluidas aquellas variables de reconocido valor pronóstico, así como otras que en el análisis univariado mostraron asociación significativa $(\mathrm{p}<$ 0.05) con la variable respuesta. Se trabajó con una confiabilidad del $95 \%$.

\section{Resultados}

La media de la edad global fue de 67,8 años siendo la mayoría del sexo masculino $(70,7 \%)$ y color de la piel blanca (82,7\%). Los pacientes fueron divididos en dos grupos según hubiesen fallecido o no durante el ingreso. Al comparar estos grupos la media de la edad fue significativamente mayor en el grupo de los fallecidos y no presentaron diferencias significativas en cuanto al sexo, color de la piel y antecedentes patológicos personales (Ver Tabla 1).

Los resultados de las comparaciones entre ambos grupos en cuanto a variables hemodinámicas al ingreso y topografía del IAM se muestran en la Tabla 2. Al comparar las variables hemodinámicas al ingreso y la topografía del IAM, el hallazgo más relevante es la fuerte asociación de la clasificación Killip Kimball >l con el grupo de los fallecidos (Ver Tabla 2). De acuerdo a la distribución de los pacientes según complementarios, tratamiento trombolítico y complicaciones, la media de la glucemia, creatinina e índice leucoglucémico fue significativamente mayor en el grupo de los fallecidos (Ver Tabla 3). 
El análisis multivariado de regresión logística binaria (Ver Tabla 4), identificó como factores pronósticos de mortalidad a las variables: creatinina sérica, tratamiento de reperfusión coronaria y la clasificación Killip y Kimball> I $(p<0.05)$.

\section{Discusión}

Está demostrada a nivel mundial la alta incidencia del IAMCEST, varios estudios coinciden con los resultados de esta serie en la cual el sexo masculino predominó y los pacientes mayores de 65 años presentaron más probabilidad de morir5,13-15.

Granger et al. ${ }^{16}$ y Morrow y et al. ${ }^{17}$ en dos relevantes estudios reportan diversos antecedentes patológicos personales como factores asociados a mortalidad intrahospitalaria en pacientes con IAM tales como hipertensión arterial, diabetes mellitus, angina, la enfermedad vascular periférica, infarto de miocardio previo, insuficiencia renal, ictus y fibrilación atrial. En la presente investigación ninguno de los antecedentes analizados estuvo asociado al estado al egreso, sin embargo, esto no indica que sean clínicamente despreciables, sino que con los datos disponibles para el período analizado no existe evidencia concluyente de que estén asociados a la mortalidad intrahospitalaria en pacientes con IAMCEST en Villa Clara.

En este estudio la media de la tensión arterial sistólica fue significativamente inferior al ingreso en los pacientes fallecidos. Estos resultados son similares a los encontrados por Aliz-Leon et al. ${ }^{5}$ y Pérez et al. ${ }^{13}$ en 128 y 137 pacientes cubanos, respectivamente. Ello se explica por la disfunción sistólica y diastólica producida por la lesión miocárdica y el consecuente descenso del gasto cardíaco da lugar a una disminución de la perfusión sistémica y coronaria. El descenso de la perfusión exacerba la isquemia y provoca la muerte celular en la zona limítrofe del infarto y la zona alejada del miocardio; empeorando así el pronóstico del paciente ${ }^{18}$.

Los resultados de esta investigación indican que la media de la glicemia del grupo caso fue mayor que la del grupo control. El aumento durante la hiperglicemia de factores vasoconstrictores e inflamatorios contribuye al daño de la función endotelial e incrementa la producción de radicales libres con el consiguiente estrés oxidativo. Además, existe un estado de resistencia insulínica en el cual la lipólisis provoca liberación de ácidos grasos libres y estos dificultan el transporte de glucosa al interior de la célula miocárdica, además, su oxidación genera aniones superóxido ${ }^{19,20}$. También produce una alteración en el metabolismo plaquetario y cambios en los mecanismos de señalización intraplaquetaria que pueden contribuir al desarrollo de las complicaciones aterotrombóticas ${ }^{21}$.

Por su parte la asociación entre un mayor conteo leucocitario y la glicemia con la muerte intrahospitalaria por IAM está demostrada. La fusión de estos dos parámetros sencillos en uno (índice leucoglicémico) y su valor pronóstico en la evolución a corto plazo fue expuesta por primera vez por Quiroga et al. ${ }^{12}$, en posteriores trabajos ${ }^{5,22,23}$, al igual que el presente, confirman la validez pronóstica del índice leucoglucémico.

En el IAMCEST las complicaciones cardiovasculares o no, definen el pronóstico de la enfermedad durante la estadía hospitalaria. En esta serie las complicaciones hemodinámicas, eléctricas y las no cardiovasculares incrementaron la probabilidad de fallecer en aquellos pacientes que las presentaron. No existieron complicaciones hemorrágicas en la muestra estudiada, ello puede deberse fundamentalmente a la adhesión a los protocolos para el empleo de la estrategia de reperfusión coronaria en estos pacientes. Resultados que coinciden con los de Pedersen et al. ${ }^{15}$ y Pérez et al. ${ }^{13}$.

El pronóstico de mortalidad intrahospitalaria tiene naturaleza multivariante por lo que los factores identificados por la regresión logística son los más confiables. En este estudio los factores pronósticos identificados fueron los valores de creatinina sérica, el tratamiento de reperfusión coronaria y la clasificación Killip y Kimball mayor que I.

La afección renal en los pacientes con enfermedad cardiovascular, confiere un carácter pronóstico y un incremento del riesgo de complicaciones. La insuficiencia cardíaca congestiva, los estados hipercatabólicos, los fármacos y tratamientos prescritos (contrastes, inhibidores de la enzima convertidora de angiotensina, antagonistas de los receptores IIb/IIIa) y el deterioro de la función renal provocan un pronóstico desfavorable en la evolución de los pacientes con IAMCEST ${ }^{17-19}$ y se reflejan en un incremento en los valores séricos de creatinina. Ello explica la relación directa encontrada en este estudio entre las cifras séricas de creatinina y el riesgo de muerte. 


\section{SEPTIEMRBRE - DIGIEMRBRE}

Los tratamientos de reperfusión coronaria modificaron la historia natural del IAMCEST al reducir de manera significativa la morbilidad y mortalidad. Esta afirmación está respaldada por investigaciones que indican una reducción en un $25 \%$ de la mortalidad a corto plazo de los pacientes que reciben trombolisis y por tanto un incremento del riesgo de fallecer en aquellos pacientes no tratados con esta terapia ${ }^{6}$. Luego del advenimiento y generalización de la intervención percutánea coronaria se logró un avance significativo en el manejo y pronóstico de estos pacientes ${ }^{24}$.

Los principales factores que influyen en la no realización del tratamiento trombolítico son: la demora de más de 12 horas desde el inicio de los síntomas y la edad avanzada. Entre los obstáculos para el inicio del tratamiento en ancianos con IAMCEST se cuentan el retraso prolongado de la solicitud de asistencia médica, menor incidencia de molestias isquémicas y mayor incidencia de síntomas atípicos, enfermedades concomitantes y hallazgos no diagnósticos en el electrocardiograma ${ }^{4}$.

La clasificación de Killip y Kimball es el resultado de un estudio" en el que se describe la evolución de 250 pacientes con IAM en función de la presencia o ausencia de hallazgos físicos que sugirieran disfunción ventricular, diferenciando cuatro clases (I, II, III y IV) para las cuales la mortalidad intrahospitalaria fue del $6,17,38$ y $81 \%$, respectivamente. El valor pronóstico de esta escala se ha demostrado en múltiples investigaciones $5,13,15$ que, al igual que el presente estudio, han obtenido como resultado que las clases mayor que I se asocian a una mayor probabilidad de fallecer en pacientes con IAMCEST.

Varios autores han estudiado los factores pronósticos de mortalidad intrahospitalaria en los pacientes con IAMCEST con resultados variables. Pérez et al. ${ }^{13}$ señalan como factor predictivo de mortalidad intrahospitalaria a la edad de más de 65 años, la enfermedad vascular periférica, la taquicardia, la hipotensión, la clase IV en la clasificación de KillipKimball y reportan al tabaquismo como un factor protector. León-Aliz et al. ${ }^{5}$ concluye en su estudio que la edad mayor a 75 años, la tensión arterial sistólica inferior a $100 \mathrm{~mm} \mathrm{Hg}$ y el índice leucoglicémico superior a 1,158 fueron predictores independientes de muerte y complicaciones cardiovasculares mayores.
En el importante ensayo clínico Intravenous nPA for Treatment of Infarcting Myocardium Early II (InTIME II) ${ }^{17}$ desarrollado en más de 14000 pacientes concluyeron que la edad, la presión arterial sistólica, el ritmo cardíaco, la clase Killip-Kimball > I, el infarto de topografía anterior con elevación del segmento ST, el bloqueo de rama izquierda, la diabetes mellitus, la hipertensión arterial, la angina de pecho, el peso menor de $67 \mathrm{~kg}$, y el tiempo para recibir tratamiento mayor de 4 horas fueron variables capaces de predecir independientemente la mortalidad intrahospitalaria. Por otra parte, el modelo pronóstico derivado del Global Registry of Acute Coronary Events (GRACE) ${ }^{16}$, en 11389 pacientes fueron identificados como factores pronósticos de mortalidad intrahospitalaria la clase Killip-Kimball, la presión arterial sistólica, la frecuencia cardíaca, la edad, la creatinina, la desviación del segmento ST, las enzimas de necrosis miocárdica aumentadas y el paro cardíaco previo al ingreso.

Por su parte, Perdersen et al..$^{15}$ en un estudio danés con 2804 pacientes en el año 2014, encuentraron a la edad, el antecedente de diabetes mellitus y de insuficiencia cardíaca, el grado de la escala Thrombolysis In Myocardial Infarction de flujo coronario de 0 a 1 después de la intervención percutánea coronaria, la clase Killip y Kimball mayor que I y el tamaño del vaso coronario afectado como factores pronósticos de mortalidad intrahospitalaria.

Este estudio tiene como limitaciones las propias de un diseño retrospectivo, la muestra estudiada es pequeña al tratarse de la experiencia de una sóla institución, además de que no se contó con la determinación de valiosos marcadores biológicos (troponinas, proteína C reactiva, interleucinas, péptido natriurético, etc.) y la realización de la intervención coronaria percutánea primaria por no estar disponibles en el Hospital Arnaldo Milián Castro. Tampoco se dispuso del tiempo entre el comienzo de los síntomas y el inicio de la terapia de reperfusión coronaria, sin dudas un dato valioso para el pronóstico de estos pacientes y a su vez, fácil de registrar en las historias clínicas. No obstante, los factores pronósticos encontrados se ajustan a la realidad asistencial villaclareña por lo que pueden ser fácilmente empleados en la práctica clínica diaria. Este reporte sirve de base para la realización de estudios posteriores que incluyan a un mayor número de pacientes en un período más largo de 
tiempo y que confirmen el valor pronóstico de los factores identificados en los pacientes del territorio.

\section{Conclusiones}

Los niveles séricos de creatinina, el tratamiento de reperfusión coronaria y la clasificación Killip y Kimball >l constituyeron los factores pronósticos de mortalidad intrahospitalaria en los pacientes villaclareños con IAMCEST. Su identificación temprana podría indicar qué pacientes necesitan un seguimiento más estrecho durante su estadía hospitalaria con el fin de mejorar su pronóstico. Estos resultados indican que medidas encaminadas a lograr un mayor índice de reperfusión coronaria en el manejo de emergencia puede disminuir la mortalidad en este grupo de estos pacientes.

\section{Consideraciones ÉtICAS}

La realización del estudio fue aprobada por el comité de ética de la institución. Los datos personales de los pacientes no fueron publicados y se siguieron los principios establecidos en la Declaración de Helsinki.

\section{Financiación}

La financiación del proyecto se realizó con recursos propios.

\section{CONFLICTO DE INTERESES}

Los autores declaran no tener conflictos de interés.

\section{Referencias Bibliográficas}

1. Roth GA, Johnson C, Abajobir A, Abd-Allah F, Abera SF, Abyu G, et al. Global, Regional, and National Burden of Cardiovascular Diseases for 10 Causes, 1990 to 2015. J Am Coll Cardiol. 2017;70(1):1-25

2. TERIMA Group of Investigators. TERIMA-2: national extension of thrombolytic treatment with recombinant streptokinase in acute myocardial infarct in Cuba. Thromb Haemost. 2000;84(6):94954.

3. Ministerio de Salud Pública, Dirección de Registros Médicos y Estadísticas en Salud. Anuario Estadístico de Salud 2016. La Habana: Ministerio de Salud Pública de Cuba; 2017.

4. Mega J, Morrow D. Infarto de miocardio con elevación del ST: tratamiento. In: Braunwald E, ed. by. Tratado de Cardiología: Texto de Medicina Cardiovascular. España: Elsevier; 2016. p. 1055-147.

5. León-Aliz E, Moreno-Martínez FL, Pérez-Fernández GA, VegaFleites LF, Rabassa-López-Calleja MA. Índice leuco-glucémico como marcador pronóstico de la evolución intrahospitalaria en pacientes con infarto agudo de miocardio con elevación del ST. Clin Invest Arterioscl. 2014;26(4):168-75.

6. Novo LE, Gómez YdC, Milord Y, Ramos HL. Caracterización de pacientes con infarto miocárdico con elevación del ST complicados no trombolizados. Acta Médica del Centro. 2014;8(1):29-36.

7. Bustaque Y, Pérez L. Comportamiento del lipidograma en pacientes post-infarto agudo del miocardio. Acta Médica del Centro. 2011;5(3):56.

8. González K, Expósito LR, Lugo L, Rodríguez A, Leal JL. Comportamiento del infarto agudo del miocardio en una Sala de Cuidados Intensivos Cardiológicos en el período de un año. Acta Médica del Centro. 2011;5(3):31-2.

9. Thygesen K, Alpert JS, Jaffe AS, Simoons ML, Chaitman BR, White HD, et al. Third universal definition of myocardial infarction. J Am Coll Cardiol. 2012;60(16):1581-98.

10. Villa JJ, Pallás JMA. Métodos de investigación clínica y epidemiológica. 4th ed. España: Elsevier; 2012.

11. Killip T, Kimball JT. Treatment of myocardial infarction in a coronary care unit: a two year experience with 250 patients. Am J Cardiol. 1967;20(4):457-64.

12. Castro W, Conci E, Zelaya F, Isa M, Pacheco G, Sala J, et al. Estratificación del riesgo en el infarto agudo de miocardio según el índice leucoglucémico. ¿El Killip-Kimball de laboratorio?. Rev Fed Arg Cardiol. 2010;39(1):29-34.

13. Pérez JEL, Jiménez RR, Negret AL, Mena MG, Delgado MD, Fernández RV, et al. Factores predictivos de mortalidad en pacientes con infarto agudo del miocardio con elevación del ST. Rev Cubana Cardiol Cir Cardiovasc. 2012;16(4):417-23.

14. Bernat I, Horak D, Stasek J, Mates M, Pesek J, Ostadal P, et al. ST-segment elevation myocardial infarction treated by radial or femoral approach in a multicenter randomized clinical trial: the STEMI-RADIAL trial. J Am Coll Cardiol. 2014;63(10):964-72.

15. Pedersen F, Butrymovich V, Kelbæk H, Wachtell K, Helqvist S, Kastrup J, et al. Short-and long-term cause of death in patients treated with primary PCI for STEMI. J Am Coll Cardiol. 2014;64(20):2101-8.

16. Granger CB, Goldberg RJ, Dabbous O, Pieper KS, Eagle KA, Cannon CP, et al. Predictors of hospital mortality in the global registry of acute coronary events. Arch Intern Med. 2003;163:2345-53.

17. Morrow DA, Antman EM, Charlesworth A, Cairns R, Murphy SA, de Lemos JA, et al. TIMI risk score for ST-elevation myocardial infarction: a convenient, bedside, clinical score for risk assessment at presentation. Circulation. 2000;102(17):2031-7.

18. Reynolds HR, Hochman JS. Cardiogenic Shock Current Concepts and Improving Outcomes. Circulation. 2008;117:686-97.

19. Angeli F, Reboldi G, Poltronieri C, Lazzari L, Sordi M, Garofoli $\mathrm{M}$, et al. Hyperglycemia in acute coronary syndromes: from mechanisms to prognostic implications. Ther Adv Cardiovasc Dis. 2015;9(6):412-24.

20. Koracevic G, Vasiljevic S, Velicković-Radovanović R, Sakac D, Obradovic S, Damjanovic M, et al. Stress hyperglycemia in acute myocardial infarction. Vojnosanit Pregl. 2014;71(9):85869.

21. Puig Domingo M. Función plaquetaria e hiperglucemia en el síndrome coronario. Rev Esp Card. 2014;67:3-5.

22. Hirschson Prado A, Higa C, Merlo P, Dominé E, Blanco P, Vázquez G, et al. Valor pronóstico del índice leucoglucémico en el infarto agudo de miocardio. Resultados del Registro Multicéntrico SCAR. Rev Fed Arg Cardiol. 2014;82:500-5.

23. Benítez RE, Morales AM, Hernández LM, Sánchez PA, Herrera Y, Rivera EM. Hemoglobina glucosilada e índice leucoglucémico como determinaciones pronósticas en el síndrome coronario agudo. CorSalud. 2016;8(3):153-63.

24. Boersma E. The Primary Coronary Angioplasty vs. Thrombolysis (PCAT)-2 Trialists' Collaborative Group. Does time matter? A pooled analysis of randomized clinical trials comparing primary percutaneous coronary intervention and in-hospital fibrinolysis in acute myocardial infarction patients. Eur Heart J. 2006;27(7):779-88. 
Tabla-1: Distribución de los pacientes según variables epidemiológicas y antecedentes patológicos personales. Hospital Arnaldo Milián Castro.2015

\begin{tabular}{|c|c|c|c|c|c|}
\hline Variables & $\begin{array}{l}\text { Total } \\
\mathrm{N}=75\end{array}$ & $\begin{array}{c}\text { Grupo Caso } \\
n=15\end{array}$ & $\begin{array}{l}\text { Grupo Control } \\
\qquad n=60\end{array}$ & $P$ & OR (IC 95\%) \\
\hline Edad $^{a}$ & $67.8 \pm 11.91$ & $74.13 \pm 7.80$ & $65.82 \pm 12.22$ & $0.015^{b}$ & \\
\hline$<65$ & $33(44.0)$ & $2(13.3)$ & $31(51.7)$ & & \\
\hline$\geq 65$ & $42(56.0)$ & 13(86.7) & $29(48.3)$ & $0.007^{b}$ & $6.948(1.442$ a 3.480$)$ \\
\hline \multicolumn{4}{|l|}{ Sexo(\%) } & 0.120 & \\
\hline Masculino & $53(70.7)$ & $8(53.3)$ & $45(75.0)$ & & \\
\hline Femenino & $22(29.3)$ & $7(46.7)$ & $15(25.0)$ & & \\
\hline \multicolumn{4}{|l|}{ Color de piel( $\%)$} & 0.279 & \\
\hline Blanca & $62(82.7)$ & 11(73.3) & $51(85.0)$ & & \\
\hline No blanca & 13(17.3) & $4(26.7)$ & $9(15.0)$ & & \\
\hline \multicolumn{6}{|c|}{ Antecedentes patológicos personales(\%) } \\
\hline Tabaquismo & $28(37.3)$ & $4(26.7)$ & $24(40.0)$ & 0.388 & \\
\hline Diabetes mellitus & $20(26.7)$ & $5(33.3)$ & $15(25.0)$ & 0.526 & \\
\hline Dislipidemia & $17(22.7)$ & $3(20.0)$ & $14(23.3)$ & 1.000 & \\
\hline Hipertensión Arterial & $53(70.7)$ & 13(86.7) & $40(66.7)$ & 0.205 & \\
\hline Obesidad & $17(22.7)$ & $4(26.7)$ & $13(21.7)$ & 0.734 & \\
\hline Alcoholismo & $6(8.0)$ & $1(6.7)$ & $5(8.3)$ & 1.000 & \\
\hline $\begin{array}{l}\text { Enfermedad vascular } \\
\text { periférica }\end{array}$ & $5(6.7)$ & $3(20.0)$ & $2(3.3)$ & 0.052 & \\
\hline IAM previo & $16(21.3)$ & $6(40.0)$ & $10(16.7)$ & 0.074 & \\
\hline
\end{tabular}

Fuente: Historias Clínicas aResultado expresado como media $\pm D E$ bEstadísticamente significativo

Tabla-2: Distribución de los pacientes según variableshemodinámicas al ingreso y topografía del IAM.

\begin{tabular}{|c|c|c|c|c|c|}
\hline Variables & $\begin{array}{l}\text { Total } \\
N=75\end{array}$ & $\begin{array}{c}\text { Grupo Caso } \\
n=15\end{array}$ & $\begin{array}{l}\text { Grupo Control } \\
n=60\end{array}$ & $\mathbf{P}$ & OR (IC 95\%) \\
\hline Tensión arterial sistólica (mmHg)a & $122.0 \pm 22.7$ & $102.6 \pm 37.8$ & \multicolumn{2}{|c|}{$126.8 \pm 22.5$} & $0.009^{b}$ \\
\hline Tensión arterial diastólica(mmHg)a & $77.7 \pm 15.1$ & $70.6 \pm 20.1$ & \multicolumn{2}{|c|}{$79.5 \pm 13.2$} & 0.072 \\
\hline Frecuencia cardíaca (latidos/min)a & $80.9 \pm 20.0$ & $88.2 \pm 29.8$ & \multicolumn{2}{|c|}{$79.13 \pm 16.6$} & 0.516 \\
\hline \multicolumn{5}{|l|}{ Killip y Kimball(\%) } & $0.000^{\mathrm{b}}$ \\
\hline I & $41(54.7)$ & $2(13.3)$ & \multicolumn{2}{|c|}{$39(65.0)$} & \\
\hline$>1$ & $34(45.3)$ & 13(86.7) & \multicolumn{2}{|c|}{$21(35.0)$} & $0.000^{\mathrm{b}}$ \\
\hline \multicolumn{5}{|l|}{ Topografía del IAM(\%) } & 0.356 \\
\hline Anterior & $37(49.3)$ & $9(60.0)$ & \multicolumn{2}{|c|}{$28(46.7)$} & \\
\hline Inferior & $38(50.7)$ & $6(40.0)$ & \multicolumn{2}{|c|}{$32(53.3)$} & \\
\hline
\end{tabular}

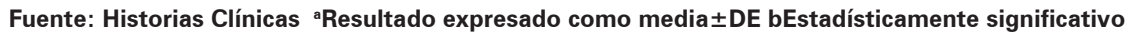


Tabla-3: Distribución de los pacientes según complementarios, tratamiento trombolítico y complicaciones.

\begin{tabular}{|c|c|c|c|c|c|}
\hline Variables & $\begin{array}{l}\text { Total } \\
\mathrm{N}=75\end{array}$ & $\begin{array}{c}\text { Grupo Caso } \\
n=15\end{array}$ & $\begin{array}{c}\text { Grupo Control } \\
\qquad n=60\end{array}$ & $p$ & OR (IC 95\%) \\
\hline \multicolumn{6}{|l|}{ Complementarios } \\
\hline Hematócrito & $0.40 \pm 0.05$ & $0.38 \pm 0.04$ & $0.40 \pm 0.05$ & 0.108 & \\
\hline Glicemiaa $^{a}$ & $9.69 \pm 6.74$ & $14.2 \pm 10.3$ & $8.55 \pm 4.99$ & $0.012^{b}$ & \\
\hline Leucocitos $^{a}$ & $11.11 \pm 2.43$ & $11.4 \pm 2.25$ & $11.0 \pm 2.48$ & 0.289 & \\
\hline Neutrófilos ${ }^{a}$ & $0.72 \pm 0.10$ & $0.74 \pm 0.08$ & $0.72 \pm 0.10$ & 0.412 & \\
\hline Linfocitos $^{a}$ & $0.25 \pm 0.09$ & $0.23 \pm 0.08$ & $0.26 \pm 0.09$ & 0.367 & \\
\hline Creatinina $^{a}$ & $102.4 \pm 51.8$ & $145.5 \pm 85.0$ & $91.6 \pm 32.7$ & $0.005^{b}$ & \\
\hline Pico de $\mathrm{CK}^{\mathrm{a}}$ & $1785.5 \pm 1676.1$ & $2044.8 \pm 1837.1$ & $1720.7 \pm 1643.5$ & 0.479 & \\
\hline Índice leucoglicémicoa & $1.975 \pm 1.599$ & $3.097 \pm 2.666$ & $1.695 \pm 1.059$ & $0.011^{\mathrm{b}}$ & \\
\hline Trombolisis(\%) & $45(60.0)$ & $5(33.3)$ & $40(66.7)$ & $0.018^{b}$ & $0.25(0.075$ a 0.830$)$ \\
\hline \multicolumn{6}{|l|}{ Complicaciones(\%) } \\
\hline Eléctricas & $25(33.3)$ & 10(66.7) & $15(25.0)$ & $0.002^{b}$ & $\begin{array}{c}6.00(1.767 \text { a } \\
20.369) \\
\end{array}$ \\
\hline Hemodinámicas & $30(40.0)$ & $15(100)$ & $15(25.0)$ & $0.000^{\mathrm{b}}$ & $2.00(1.398$ a 2.860$)$ \\
\hline Isquémicas & $3(4.0)$ & $0(0.0)$ & $3(5.0)$ & 0.377 & \\
\hline Trombóticas & $1(1.3)$ & $1(6.7)$ & $0(0.0)$ & 0.200 & \\
\hline Inflamatorias & $1(1.3)$ & $1(6.7)$ & $0(0.0)$ & 0.200 & \\
\hline Otras & $9(12.0)$ & $5(33.3)$ & $4(6.7)$ & $0.016^{b}$ & $\begin{array}{c}7.00(1.598 \text { a } \\
30.657)\end{array}$ \\
\hline
\end{tabular}

Fuente: Historias Clínicas aResultado expresado como media $\pm D E$ 'Estadísticamente significativo

Tabla 4: Variables pronósticas de mortalidad intrahospitalaria en pacientes con IAMCEST.

\begin{tabular}{|l|c|c|c|c|c|}
\hline \multicolumn{1}{|c|}{ Variables } & Coeficiente de regresión & Error estándar & $\mathbf{X}^{\mathbf{2}}$ de Wald & $\boldsymbol{P}$ & OR (IC 95\%) \\
\hline Creatinina sérica & 0.013 & 0.007 & 3.854 & 0.049 & $1.013(1.010$ a 1.027) \\
\hline $\begin{array}{l}\text { Tratamiento de reperfusión } \\
\text { coronaria }\end{array}$ & -1.580 & 0.734 & 4.632 & 0.031 & $0.206(0.049$ a 0.868) \\
\hline Killip y Kimball> I & 2.118 & 0.870 & 5.926 & 0.015 & $8.311(1.511$ a 45.720) \\
\hline Constante & -3.424 & 0.969 & 12.480 & 0.000 & 0.033 \\
\hline
\end{tabular}

Fuente: Procesamiento estadístico 